\title{
The PREFER (Polarization REsearch for Fusion Experiments and Reactors) - collaboration: purposes and present status
}

\author{
G. Ciullo*@, L. Barion and M. Statera \\ Dipartimento di Fisica e Scienze della Terra, Università degli Studi di Ferrara and Istituto \\ Nazionale di Fisica Nucleare - sezione di Ferrara (INFN-Fe) - I-44122 Ferrara, Italy \\ $\sharp$ now at the Istituto Nazionale di Fisica Nucleare - LASA - Sezione di Milano - I- 20090 \\ ${ }^{\circledR}$ E-mail: ciullo@fe.infn.it \\ R. Engels ${ }^{\circledR}$, K. Grigoryev and L. Huxold ${ }^{\dagger}$ \\ Institut für Kernphysik (IKP), Forschungszentrum Jülich (FZJ), - 52428 Jülich, Germany \\ ${ }^{\circledR}$ E-mail: r.w.engels@fz-juelich.de
}

\section{Büscher ${ }^{\dagger}, @$ and A. Hützen ${ }^{\dagger}$}

Peter Grünberg Institut (PGI) Forschungszentrum Jülich (FZJ), - 52428 Jülich, Germany

$\dagger$ also Institut für Laser- und Plasmaphysik Heinrich-Heine-Universität Düsseldorf,

(ILPP-HHUD) - D-40225 Düsseldorf, Germany

${ }^{\circledR}$ E-mail: m.buescher@fz-juelich.de

V. D. Fotev, K. A. Ivshin, E. N. Komarov, L. M. Kotchenda, P. V. Kravchenko@ ${ }^{\circledR}$, P. A. Kravtsov, S. G. Sherman, A. N. Solovev, I. N. Solovyev, V. A. Trofimov, A. A. Vasilyev and M. E. Vznuzdaev

Petersburg Nuclear Physics Instituted (PNPI) named by B. P. Kostantinov of the National

Research Center "Kurchatov" Institute (NRC-KI)-Ru-188300 Gatchina, Russia

${ }^{\circledR} E$-mail: kravchenko_pv@inp.nsk.su

D. Toporkov ${ }^{\S, @ ~, ~ I . ~ A . ~ R a c h e k ~ a n d ~ Y u . ~ V . ~ S h e s t a k o v ~}{ }^{\S}$

Budker Institute of Nuclear Physics (BINP)- ru-630090 Novosibirsk, Russia

$\S$ also at Novosibirsk State University - ru-630090 Novosibirsk, Russia

${ }^{\circledR}$ E-mail: D.K.Toporkov@inp.nsk.su

\section{T. P. Rakitzis ${ }^{@}$, D. Sofikitis, C. S. Kannis ${ }^{\ddagger}$ and G. K. Boulogiannis}

Department of Physics, University of Crete and Institute of Electronic Structure and Laser(IESL), Foundation for Research and Technology Hellas (FORTH) -71110

Heraklion-Crete, Grece

$\ddagger$ also RWTH-AAchen University - 52062 Aachen, Germany

${ }^{\circledR}$ E-mail: ptr@iesl.forth.gr 
The possibility to exploit spin physics in fusion science opens new impressive panorama and challenging fields to be explored. In addition to the fundamental interesting topics the PREFER collaboration is trying with synergistic efforts to make the well known behavior of spin-dependent nuclear reactions available for feasibility studies and experimental tests for fusion programs. In this contribution the main challenges of the different collaborating groups will be put in evidence, and recent developments with the respective references will be provided.

The 18th International Workshop on Polarized Sources, Targets, and Polarimetry, PSTP2019 23-27 September, 2019

Knoxville, Tennessee

${ }^{*}$ Speaker. 


\section{Introduction}

The recent climate and planetary scenario is pushing, at present, the fusion programs and diffusing worldwide the consciousness that the alternative energy resources, moderating the $\mathrm{CO}_{2}$ concentration in the atmosphere and in ocean, are proved to be not enough for the present human population, which has still a growing trend, and an increasing request at least of primary energies.

The impelling demand of energy under environmental and safety concerns is leading to increased emphasis on renewable energy sources, which need the support in the future of nuclear fusion energy production. The fusion program is in the plan, but still not at the proper phase for energy production, and still with very high efforts and costs.

The idea of using spin-polarized fuel for fusion was proposed long ago [1]. The promising advantages, which relax the engineering constrains and therefore the costs of fusion reactors and facilities, were discussed, but some disadvantages have overshadowed the path of the researches.

Exploring the possibilities of fusion with nuclear polarized fuel involves various fields of investigations, technologies, researches and developments. The improvements during the last fifty years on the manipulation and the operation of polarized nuclear targets, recover the hope of pursuing the challenging task of its use for the social, climate and peaceful project of energy production from nuclear fusion: deuterium, which is the main element for the most accessible fusion reactions, can be extracted from water which is worldwide available.

The PREFER collaboration is born for common interests and deals in these challenging topics.

In the following we will report on the status of these efforts, emphasizing in bold the main groups, or where the research is performed, and in brackets the orbiting groups, which directly collaborate on the topics. The groups will be indicated by using the acronyms of the affiliations of the author list. The challenges undertaken by each group can be summarized as follows:

PNPI (IKP-FZJ/INFN-Fe): D-D spin dependent cross section studies and measurements.

IKP-FZJ (PNPI/INFN-Fe): Production of polarized fuel from the recombination of polarized deuterium atoms from an atomic beam source (pABS) and its handling.

BINP (HHUD-ILPP/IKP-FZJ): Hyper-polarized molecules from a polarized Molecular Beam Source (pMBS).

IESL-FORTH (PGI-FZJ/HHUD-ILPP): Production of polarized beams from laser quantum beat excitation and post UV photo-fragmentation.

PGI-FZJ (ILPP-HHUD): Laser-induced plasmas: production of polarized ion beams, acceleration and fusion tests.

Before presenting the details of the different fields under investigation, we would like to remind the state-of-the-art of nuclear fusion with polarized fuel, and the open questions, which stimulate the aforementioned challenges.

\section{Nuclear fusion with polarized fuel}

The exploitation of polarized fuel for nuclear fusion a priori can provide very interesting advantages, but there are also still open questions. 
It is useful to summarize the advantages as follows:

Enhancement of fusion cross-section. The enhancement of fusion cross-sections is theoretically well understood for spin 1-spin 1/2 interacting particles, e. g. for the deuterium (D)-tritium (T), or D- ${ }^{3} \mathrm{He}$, fusion reactions. However the fusion cross-section for spin 1-spin 1 interacting particles (D-D) it is still not fully understood.

Control of angular distributions of the reaction products. Well known neutron angular distributions allow to concentrate the problem of neutron wall bombardments, radioactivity activation, and degradation of materials and components, in a limited space of the surrounding of the fusion volume. And concentration of the neutrons can help for further fuel breading via the $\mathrm{n}+{ }^{6} \mathrm{Li} \rightarrow{ }^{4} \mathrm{He}+\mathrm{t}$ reaction. In addition a better confinement of charged particles in the plasma will heat it more and more, then requiring less energy from outside to maintain the fusion reactions.

Possible neutron lean reactors. Dealing with the neutron production from the D-D fusion reaction, in case of aligned spins this reaction would be prohibited in the pure S-wave approximation, but unfortunately the reaction experiences $\mathrm{S}_{-}, \mathrm{P}-$, and $\mathrm{D}-$ partial wave scattering and their interference. There is no data available for the constrains of theoretical models for the spin-dependent cross-sections. These should be obtained from controlled interactions in beam-target fusion experiments.

Anyhow the practical use, also for the well known case of spin 1- spin 1/2 interacting particles, which is the most accessible reaction for fusion, is still far away mainly due to still open questions and requirements, which we summarize here as disadvantages in the following:

Intensity (or density), purity and high polarization. The achievable intensities of polarized fuel, following the technologies implemented for polarized gas targets in nuclear physics in order of $10^{17}$ atoms s$^{-1}$, are still not in the range of the fusion requirements, $10^{22}$ atoms s${ }^{-1}$, though the purity and polarization can reach very high values.

At the contrary, for solid polarized nuclear targets purity and polarization are much lower than what is required for fusion, but the density in this case is not a problem.

But not only the production is a problem. The manipulation of the polarized fuel for its use in the fusion environment will require new technological approaches. Considering the improvements in the recent decades on the technology developed for polarized nuclear target, the engagement on this challenge could be very fruitful.

Preparation, Manipulation and Transportation. More problems have to be faced for the use of polarized fuel, its preparation, the transportation, and the proper manipulation for fusion facilities, or fusion tests.

Survival of the polarization in plasma. Once, the preparation, transportation and manipulation of polarized fuel is understood, there are still open questions on the survival of the polarization in the fusion environments.

The framework of the purposes of this researches can be organized according to the reaction generations with respect to the colliding particle energy, or the temperature of the plasma.

H. Paetz gen. Schieck summarized in various contribution the state-of-the-art of nuclear fusion with polarized fuel (often called also "Polarized Fusion" in a compact way) and in recent reviews [2,3] the advantages and the disadvantages are discussed with suggestions and mandatory requirements.

The first generation fusion reaction/reactor involves $\mathrm{D}$ and $\mathrm{T}$, as the fuel for $\mathrm{D}+\mathrm{T} \rightarrow{ }^{4} \mathrm{He}+$ 
$\mathrm{n}$ reaction. From the point of view of spin it means interacting particles with spin 1 and spin $1 / 2$ respectively. The spin-dependent behavior of the reaction was experimentally proved in 1971 [4], and the theoretical comprehension of spin-dependent reactions was already in very good shape as documented in a review in 1972 [5]. If the interacting particles have the vector spins oriented in the same direction, the cross-section will be enhanced by a factor 1.5 . A nice pictorial view of the other spin configurations and the respective angular distributions of the reaction products is presented in Ref. [6]. In the case of the D-T, reaction which proceeds via a strong S-wave scattering [3], the cross-section is strictly given by spin and momentum.

The second generation reaction involve deuterium and there are two possible branches, $\mathrm{D}+\mathrm{D} \rightarrow$ $\mathrm{T}+\mathrm{p}$ and $\mathrm{D}+\mathrm{D} \rightarrow{ }^{3} \mathrm{He}+\mathrm{n}$. Deuterium is mainly the fuel for research facilities, which are equipped for proper diagnostics and monitoring of the reactions.

In the case of the pure D-D reaction the description of the mechanism is very complicated, $\mathrm{S}-, \mathrm{P}-$ and D-wave scattering and the respective interference have to be considered, which do not allow a clear prediction on spin-dependent cross-sections in a simple picture. There are various theoretical models strongly constrained by data, which are not available in a sufficient way, or it is better to say are totally absent, to address the proper model for spin-dependent nuclear reactions.

The D-D reaction is also present, to a small amount, in the D-T first generation reactors. Therefore the comprehension of its spin dependence should contribute more to the fusion program and the design of reactors.

The third generation reaction, $\mathrm{D}+{ }^{3} \mathrm{He} \rightarrow{ }^{4} \mathrm{He}+\mathrm{p}$, from the point of view of spin-dependent cross-section, is equivalent to D-T. In fact, the experimental proof of the theoretical model was performed exactly on this reaction [4].

The realization of a "neutron lean" reactor requires the understanding of the D-D spin-dependent cross-section. If both interacting particles are aligned parallel to the magnetic field, the reaction, in the $\mathrm{S}$-wave approximation, is prohibited. The neutron production is suppressed in a third generation reactor.

This problem, known as Quintet Suppression Factor (QSF), the ratio between the cross-section of vector aligned deuterium interacting particles and the unpolarized cross-section, is a mandatory measurement, which involves a few-body nuclear reaction also of interest for astrophysics. There are models which instead predict an enhancement of the reaction by a factor of up to 2.5, and models which estimate this ratio close to zero, confirming the QSF [7]. The understanding of the D-D spin-dependent reaction is the basis for the future exploitation of the polarized fuel for fusion, but on itself it will provide deep insight in still open questions for nuclear reactions, fewbody systems and astrophysical questions [3].

There is one more argument which complicates the panorama on fusion prevision, which is the electron screening at the low energy of fusion reaction. The electron screening is also contributing to the enhancement of cross-sections in the energy range of fusion, which then, summed to the spin-dependent enhancement of the cross-section, could play a dominant role for the chance to use nuclear fusion for energy production, not only reducing the power required for ignition and maintenance of the fusion processes, but also, as a consequence, reducing the costs of the design and the operation of the fusion reactors and facilities. 


\section{The PREFER challenging objectives: study, production and test of polarized fuel}

The impelling questions on deuterium and its presence in the first three generations of fusion reactions, make it the most interesting objective of the following studies and researches:

- The studies and investigations on spin-dependent cross-sections.

- The production of polarized deuterium fuel for feasibility studies and tests of nuclear fusion.

- Manipulation and transportation of the fuel for dedicate studies in proper facilities with ad hoc diagnostics, in order to test the polarization survival in fusion environments.

In the following the research fields, under the investigation of the collaboration, will be presented giving evidence in bold of the leading group, and in brackets the directly collaborating groups.

The D-D spin dependent cross-section studies at PNPI (IKP-FZJ, INFN-Fe).

For the case of D-D spin dependent cross-sections there are still missing data. The first proposal of studying the double polarized cross-section on D-D reactions dates back to 1969 [8]. In that time due to the so small cross-sections and the low intensity of atomic beams, in the order of $10^{11}$ atoms $\mathrm{s}^{-1}$, there were no sensible evidence on the high number of spin correlation coefficients involved in order to tune properly the theoretical model with required experimental parameters. Nowaday polarized atomic beams can reach the intensity of $10^{17}$ atoms s${ }^{-1}$, good control on the spin orientation of the nucleus, and to get high polarization values is possible.

At the PNPI, Gatchina - Russia, an experiment [7] is being commissioned, for double spin polarized D-D cross-section studies.

The experiment will use a crossed beam scheme, where $1.310^{14}$ deuterons $\mathrm{s}^{-1}$ are produced by a polarized ion source with polarization values about $P_{z}= \pm 0.8$. A polarized deuterium beam of $410^{16}$ atoms s${ }^{-1}$ with polarization values above $P_{z}= \pm 0.8$ will collide with the ions orthogonally. The estimated time for collecting sufficient statistics for the opening question of the Quintet Suppression Factor amounts to roughly three weeks.

The experiment will operate at a luminosity, that was never in hand in experiments on colliding polarized atomic beams. It allows one to measure the reaction product at any combination of spin orientation of the projectile and the target.

The ion beam energy can be set in the range of $10-100 \mathrm{keV}$, covering interesting range both for fusion and for investigation of the electron screening effects at low energy. The measurement of the angular distributions of the reaction products is fully covered by a $4 \pi$-solid angle detector around the D-D interaction volume with a typical angular resolution of 10-15 degrees.

The detector was made as a cubic structure with the inner surface covered with 576 Hamamatsu S3590 silicon PIN diodes (51 \% coverage of the solid angle). A partial number of PIN diodes were installed and the measurements of the reaction products were performed using a solid deuterium target [7].

A Lamb-shift polarimeter is installed and under commissioning for the monitoring of the polarization of both the polarized ion beam, and the polarized atomic beam [9].

Production of polarized fuel from the pABS at IKP-FZJ (PNPI, INFN-Fe)

One method to produce polarized fuel for the PREFER collaboration is thought the recombination of polarized atoms, produced by a polarized Atomic Beam Source (pABS), in order to obtain 
molecules which maintain the nuclear spin orientation (hyper-polarized molecules). Afterwards, the polarized $\mathrm{D}_{2}$ or $\mathrm{HD}$ molecules can be frozen on a cold surface to collect and store enough fuel for further fusion tests. The experience gained with polarized atomic beam sources for the production of polarized nuclear targets and their diagnostics in previous experiments like HERMES@DESY [10], and recent ones like ANKE@COSY [11], or PAX@COSY [12], will be exploited.

What was a systematic uncertainty in the atomic polarization of gaseous polarized nuclear targets, the remaining polarization of the recombined molecules in the target, come out to be a resource for fusion programs. Atomic hydrogen, or deuterium, are strongly reactive and it is not possible to bottle them, or freeze them, on a surface. The idea is to recombine the polarized atoms in molecules, but in a well defined nuclear spin orientation and "keep" them for the use in fusion studies. The purpose requires a deep investigation on atom-surface interactions, which allow a complete recombination of the injected atomic beam, and a full preservation of the nuclear polarization within the molecules.

The apparatus was built at the PNPI and then moved to the FZJ. It allows to use different materials for the coating of a recombination cells. The cell is immersed in a longitudinal magnetic field, generated by a superconducting solenoid, which can provide a magnetic holding field higher than $1 \mathrm{~T}$, to keep them in the prepared quantum state. When these molecules reach a cold surface below $10 \mathrm{~K}$ they might be accumulated as polarized ice to collect sufficient amounts for further fusion purposes.

Big progress has been done on the diagnosis of the phenomena [13]. As a results it is now proved that it is possible to produce "hyper-polarized" molecules of $\mathrm{H}_{2}, \mathrm{D}_{2}$, and $\mathrm{HD}$ with a large nuclear polarization $\approx 0.8$. HD is a perfect training ground for the studies of DT molecules.

The possibility of using frozen magnetic field inside a $\mathrm{MgB}_{2}$ cylinder without any power supply, open the door to transport the polarized ice for further tests in fusion facilities. The low temperatures to keep the magnetic field inside the $\mathrm{MgB}_{2}$ cylinder, can be induced by liquid helium, which will keep the deuterium ice solid at the same time. Therefore, in combination with a helium reservoir, the transportation of the polarized deuterium would be possible.

To transport the polarized ice from one place to another at very low temperatures and inside large magnetic fields a new technique is under development at the University of Ferrara, Italy [16]: a superconducting cylinder of $\mathrm{MgB}_{2}$ is able to "freeze" a magnetic field inside in the Tesla regime without any connection to a power supply. Until this cylinder is superconducting at low temperatures even the polarized molecules will stay in the solid phase. The cooling itself can be done with liquid helium from a transportable reservoir.

\section{Hyper-polarized molecules from pMBS at BINP (HHUD, IKP-FZJ)}

The idea of filtering molecules according to the nuclear spin follows the technique of the SternGerlach separation in an in-homogeneous magnetic field, used in polarized atomic beam sources. The difference is that the strength of the combined nuclear magnetic moment of a molecule compared to the one of an atom, is in the order of 600 less. Therefore the magnetic field required scales with the same factor, also having steeper field gradients. The available technology nowaday relies on superconducting magnets.

The group of Novosibisk implemented the updgrade of an existing superconducting atomic beam sources for its feasibility studies of spin separation of molecular beams. The geometry of the 
system is pushed in order to exploit the maximum gradient of field, therefore the source consist of a nozzle in an annular configuration, which allows to injected the molecular beam in proximity of the magnetic system, consisting of sextupole superconducting magnets of a cylindrical geometry. The molecules are separated according to their nuclear spin projections. The components focused fill a standard compression volume [15, 14], a standard way used for the intensity measurements. Cooling down the annular nozzle to $6.5 \mathrm{~K}$ the results confirm the MonteCarlo simulation of an expected flow in the order of $310^{12}$ molecules $\mathrm{s}^{-1}$ in the case of $\mathrm{H}_{2}$ hyper-polarized molecules.

The measured maximum intensity of the polarized $\mathrm{D}_{2}$ jet was $510^{11}$ molecules $\mathrm{s}^{-1}$. The smallness of this value compared to the intensity for $\mathrm{H}_{2}\left(310^{12}\right.$ molecules s$\left.{ }^{-1}\right)$ is due to the lower nuclear magnetic moment of $\mathrm{D}_{2}$ in comparison with that of the $\mathrm{H}_{2}$ molecules. The test on the existing adapted source is promising and allows to perform tests in order to refine the parameter in order to design a polarized molecular beam source. The length of the deflecting field is almost arbitrary and should be optimized to get a proper separation of molecules. The use of capillaries instead of the annular nozzle can be optimized for higher intensity and a more directional beam from the nozzle. Polarized molecules could be condensed by cryo-surface, technology under development for the recombined molecules at the IKP-FZJ. It is expected that such a source could provide an intensity of polarized molecules compared with the intensity of the best atomic beam sources $10^{17}$ molecules $\mathrm{s}^{-1}$. Molecules with the projection of magnetic moment along the beam axis having the value $m_{I}=-1$ are focused towards the beam axis, and they could be eventually directed by turbomolecular pump towards the freezing surface.

Till now the study were performed on the beam intensity, but also for this apparatus it is in plan the implementation of a Lamb-shift polarimeter [9] in order to verify the polarization of the focused molecules.

Laser induced plasma: production, acceleration and fusion FZJ/HHUD (IESL-FORTH) Laser technology can be exploited for polarized ion acceleration, polarization survival and fusion test. It is a very promising tool which matches properly in the effort of the production of polarized $\mathrm{D}_{2}$ molecules, meanwhile the involved groups are tuning the tools, theories and devices for the production, preparation and manipulation of them. The possibility of producing polarized intense ions has on itself immediate use in accelerator physics with polarized beams.

Nowaday it is possible to have polarized ${ }^{3} \mathrm{He}$ gas transportable and easily manageable, the challenge to test the survival of the polarization in picosecond shots of high power lasers on solid polarized targets of gaseous high intense polarized beams, and get as a result polarized ion accelerated at energies in the order of tens of MeV.

Recently, at the PHELIX ( Petawatt High-Energy Laser for Heavy Ion EXperiments) at GSI in Germany, laser-induced acceleration out from He supersonic jet beams, bombarded by very high power laser, has been demonstrated. The analysis of the ion-emission angle and the ion-energy spectra for all ion species could be extracted. The gas target with a density of about $10^{19} \mathrm{~cm}^{-3}$ was operated for ${ }^{3} \mathrm{He}$ and ${ }^{4} \mathrm{He}$ in preliminary studies with unpolarized supersonic atomic beams [17]. Tests on polarized ${ }^{3} \mathrm{He}$ are scheduled for November 2020, which will provide us direct tests of the survival of the polarization in laser beam ionization and acceleration.

The laser induced plasma technique is also under $\mathrm{R} \& \mathrm{D}$ for the production of polarized protons from $\mathrm{HCl}$ in conjunction with the topics of Quantum beat excitation of spin in binary molecules and the post UV laser ionization, with a density of about $10^{19} \mathrm{~cm}^{-3}$ [18] for experiments at Shanghai 
Institute of Optics and fine Mechanics (SIOM).

The works performed on laser-induced plasma acceleration will take also advantages of the experience on the polarization diagnosis of the IKP-FZJ group, which developed the Lamb-shift polarimeter, which will be used for the diagnosis of the accelerated polarized beam from the $\mathrm{HCl}$ [18].

Laser Quantum beat excitation and post UV -dissociation IESL-FORTH (FZJ/HHUD). The photo-dissociation of hydrogen halides ( $\mathrm{HCl}, \mathrm{HBr}$ ) and deuterium iodide (DI), with circularly polarized UV laser pulses, has been shown to produce ultrahigh density spin-polarized $\mathrm{H}$ and $\mathrm{D}$ atoms, and opens the way for the production of very intense polarized proton and deuteron beams[19]. The expected density has been shown to be at least in the order of $10^{19} \mathrm{~cm}^{-3}$, which is then very promising for the fusion research, or for the polarized ion in accelerator physics, which involves also the group of HHUD and FZJ.

Studies on the measurements of the polarization of the photo-fragments can be performed by the Lamb-shift polarimeter[9], a tool already used, or in plan to be used, in the different activities of the PREFER collaboration. A new method was also implemented at IESL-FORTH, by exploiting the voltage induced by transient spin polarization in a pick-up coil, which can be operated at the high intensity of the polarized photo-fragments. The expected polarization for proton produced by photo-fragmentation of $\mathrm{HCl}$ and $\mathrm{HBr}$ follows the theoretical models of molecular photo-dissociation, within a still high uncertainties of the new method of polarization measurement, but for $\mathrm{D}$ the measured value are opposite of what the theoretical models available predict, revealing errors in the theoretical models [20]. The cooperation between the groups mentioned, and the tools and knowledge in their hand, will yield fruitful results in the exploitation of this new ultrahigh-density regime of spin-polarized $\mathrm{H}$ and $\mathrm{D}$ atoms.

\section{Acknowledgments}

The work is partially supported by the Deusche Forschungsgemeinschaft (grant no, BU 2227/1 - 1), The Russian Foundation (grant no. RSF 16-42-01009) an the "Progetto Strategico" INFN-Energia.

\section{References}

[1] R. M. Kulsrud et al., Fusion Reactor Plasma with Polarized Nuclei, Phys. Rev. Lett. 49 (1982) 1248.

[2] H. Paetz gen. Schieck, "Polarized Fusion": New Aspects of an Old Project, Few-Body Syst. 54 (2013) 2159.

[3] H. Paetz gen. Schieck, Spin Physics and Polarized Fusion: Where We Stand in Nuclear Fusion with Polarized Fuel, Series: Springer Proceedings in Physics 187 (2016) 15.

[4] Ch. Leemann et al., Investigation of the ${ }^{3} \overrightarrow{\mathrm{He}}(\vec{d}, p)^{4}$ He Reaction with Polarized Beam and Target at 430 keV, Ann. Phys. 66 (1971) 810.

[5] G.G. Ohlsen, Polarization transfer and spin correlation experiments in nuclear physics, Rep. Progr. Phys. 35 (1972) 717.

[6] R. Engels et al., Advantages of Nuclear Fusion with Polarized Fuel, in proceeding of the $23^{\text {rd }}$ International Spin Physics Symposium - Spin 2018, PoS 346 PoS (SP IN2018) 1762018. 
[7] P. V. Kravchenko et al., Status of the double polarized D-D fusione esperiment, in proceeding of the $23^{\text {rd }}$ International Spin Physics Symposium - Spin 2018 PoS 346 P OS (SP IN2018) 1772018.

[8] B. P. Adyasevich, D. E. Fomenko, Analysis of investigation of the Reaction D $(d, p) T$ with polarized deuterons, Sov. J. Nucl. Phys. 9 (1969) 167.

[9] L. Huxold et al., A Lamb-Shift Polarimeter for $\overrightarrow{H_{2}}$ and $\overrightarrow{D_{2}}$ Molecules, in proceeding of the $23^{\text {rd }}$ International Spin Physics Symposium - Spin 2018 PoS 346 P OS (SP IN2018) 1052018.

[10] A. Airapetian et al., The HERMES polarized hydrogen and deuterium gas target in the HERA electron storage ring, NIM A $\mathbf{5 4 0}$ (2005) 68.

[11] M. Mikirtychyants et al., The polarized $H$ and D atomic beam source for ANKE at COSY-Jülich, NIM A 721 (2013) 83.

[12] P. Lenisa et al., Low-energy spin-physics experiments with polarized beams and targets at the COSY storage ring, EPJ Tech. Instr. 6 (2019) Art. Nr. 2.

[13] R. Engels et al., Production of hyper-polarized $\mathrm{H}_{2}$ Molecules from $\mathrm{H}$ atoms in gas-storage cells, Phys. Rev. Lett. 115 (2015) 113007.

[14] D. K. Toporkov et al., Status of the Polarized Molecular Source, in proceeding of the $23^{\text {rd }}$ International Spin Physics Symposium - Spin 2018, PoS 346 P OS (SP IN2018) 178 (2018).

[15] Yu. V. Shestakov et al., Nuclear-polarized Hydrogen/Deuterium molecular source, Phys. Part. Nucl. 50 (2019) 513.

[16] M. Statera et al., A bulk superconducting $M g B_{2}$ cylinder for holding transversely polarized targets, in proceeding of the $23^{\text {rd }}$ International Spin Physics Symposium - Spin 2018, PoS 346 POS ( SPIN2018) 1122018.

[17] I. Engin et al., Laser-induced acceleration of Helium ions from unpolarized targets, Plasm. Phys. Contr. Fusi 61 (2019) 115012.

[18] A. Hützen et al., Polarized proton beams from laser-induced plasmas, High Pow. Las. Sci. Eng. 7 (2019) Art. Nr. e16.

[19] D. Sofikitis et al., Ultrahigh-Density Spin-Polarized H and D Observed via Magnetization Quantum Beats, Phys. Rev. Lett. 121 (2018) Art. Nr. 083001.

[20] D. Sofikitis et al., Photo-fragment spin-polarization measurements via magnetization quantum beats: dynamics of DI photo-dissociation, Phys. Chem. Chem. Phys. 21 (2019) 14000. 